SCIREA Journal of Hydraulic Engineering http://www.scirea.org/journal/Hydraulic

August 29, 2021

Volume 4, Issue 2, April 2021

\title{
Comparative study on flow pattern character of pumping station forebay before and after reconstruction and simulation analysis on energy conservation and consumption reduction
}

\section{Gong Chengyong, Zeng Yongliang, Li Rennian, Ma Xijin}

School of Energy and Power Engineering, Lanzhou University of Technology, China.

\begin{abstract}
To explore the influence of the shape optimization of the forebay on energy conservation and consumption reduction of the pumping station, and analyze the mechanism of energy conservation and consumption reduction of the reconstruction of the pumping station forebay, the third pumping station forebay of Yanhuanding Water Station Water Hub was taken as the research object to compare and analyze the flow pattern character before and after reconstruction. The standard k- $\varepsilon$ turbulence model equation and the segregated solver were used to solve the discrete equations, and then the flow pattern character of the pumping station forebay before and after transformation was numerically simulated. The results indicated that the changes in the shape and size of the pumping station forebay had an impact on the loss of energy consumption. There was less eddy in the front inflow forebay, and the uniformity of the axial velocity distribution was $50.5 \%$, which was better than that of the lateral inlet $(46.9 \%)$. The energy dissipation rate in the forebay tended to be stable with the running time, and the energy consumption of the forebay got close to $492.81 \mathrm{~W} / \mathrm{m}^{2}$, which
\end{abstract}


was lower than that of the lateral inflow forebay $\left(608.13 \mathrm{~W} / \mathrm{m}^{2}\right)$. The energy-saving effect of the front inflow forebay was better than that of the lateral inflow forebay, and the flow pattern of the front inflow forebay was better than that of the lateral inflow forebay.

Keywords: Pumping station forebay, Flow pattern character, Uniformity of velocity ,Energy conservation and consumption reduction, Numerical simulation

\section{Introduction}

To achieve the requirements for the strategy of sustainable development, the policy restrictions for China's energy conservation and consumption reduction have increased, and energy conservation and consumption reduction have become increasingly important to social development. Based on the concept of energy conservation and consumption reduction, it is beneficial to improve the economic benefits of pumping stations by reasonably improving their working efficiency and reducing $\operatorname{costs}^{[1]}$. The upgrading and reconstruction of pumping stations involve pumping station unit equipment and matching water transmission system buildings. Water transmission system reconstruction aims to improve water flow conditions, reduce hydraulic loss, and ultimately improve the efficiency of pumping stations. The pumping station forebay is the main building in a stair pump station, with a complex flow pattern and relatively concentrated hydraulic loss. Therefore, the study on energy conservation and consumption reduction of a pump station forebay plays a vital role in saving energy and reducing consumption of the whole pump station.

Many scholars at home and abroad have carried out related studies on the flow pattern of the pumping station forebay. The research methods mainly include numerical simulation and model test, and the research objects are mainly the flow pattern modifying measures for the forebay. By adopting computational fluid dynamics, $\mathrm{Xu}$ Bo et al. conducted a numerical simulation on the flow pattern of the forebay of the sluice-station incorporation pump station, and then studied the flow pattern modifying effect of the open-hole diversion piers ${ }^{[2]}$. Jiang Wen et al. used the numerical simulation method to study the flow pattern modifying measures in order to improve the adverse flow patterns such as deflection, backflow, and suction vortex caused by curved flow in the pumping station forebay ${ }^{[3]}$. Tong Hongwei et al. adopted a physical model test to study the flow pattern of regulating reservoirs in a typical 
urban pumping station, and proposed corresponding flow pattern modifying measures ${ }^{[4]}$. As for large-span forebays, the combination of numerical simulation and physical model test was used to study the flow pattern modifying effect of the herringbone composite divergence piers $^{[5]}$. In this paper, starting from the perspective of energy conservation and consumption reduction, the numerical simulation method was employed to study the impact of the shape and size of the forebay before and after reconstruction on the flow pattern character, judge the change of energy consumption loss before and after the reconstruction, and ultimately explore the factors affecting the energy consumption loss.

\section{Project profile}

The research object was the forebay of the third pump station of Yanhuanding Water Lifting Hub. The designed water level elevation is $1303.41 \mathrm{~m}$, the designed flow rate is $9.62 \mathrm{~m}^{3} / \mathrm{s}$, the total head of the pump is $64 \mathrm{~m}$, and the net head is $54.63 \mathrm{~m}$. Before the reconstruction, the pumping station forebay was a lateral inflow forebay, and it was designed to install 8 units. The designed flow rate of Unit 1 was $0.88 \mathrm{~m}^{3} / \mathrm{s}$, and that of Units 2 to 8 was $1.4 \mathrm{~m} 3 / \mathrm{s}$. After the reconstruction, the shape of the forebay was the front inflow forebay, which was designed to install 6 units. The designed flow rate of Unit 1 was $0.52 \mathrm{~m}^{3} / \mathrm{s}$, and that of Unit 2 to 6 was $1.82 \mathrm{~m}^{3} / \mathrm{s}$. The structure of the forebay before and after reconstruction is shown in Figs. 1-1 and $1-2$, respectively, where $1 \# \sim 8 \#$ represent the water intake pipes of No. $1 \sim 8$ pumps.

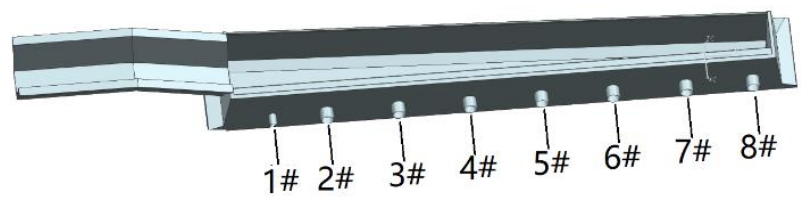

Fig. 1 Structure model of lateral inflow forebay

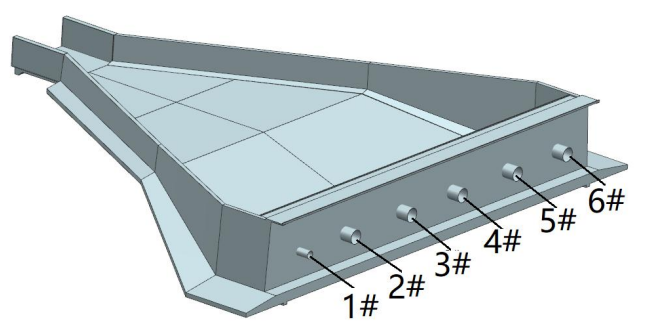

Fig. 2 Structure model of front inflow forebay

\section{Mathematical models and simulation methods}

(1) Mathematical models ${ }^{[6]}$

As the flow pattern in the forebay is complex and incompressible turbulence flow, the standard $k-\varepsilon$ turbulence model equation was chosen to describe the flow in the forebay. 
Then the CFD (Computational Fluid Dynamics) technology was used to simulate the flow pattern in the forebay under the conditions of the designed water level and designed flow in and out of the forebay. Later, the $k-\varepsilon$ model equation was composed of $k$ as turbulent kinetic energy and $\varepsilon$ as turbulent dissipation rate, and the expressions are Formula (1) and Formula (2), respectively:

$$
\frac{\partial\left(\rho_{\mathrm{m}} \varepsilon\right)}{\partial t}+\frac{\partial\left(\rho_{m} u_{j} k\right)}{\partial x_{j}}=p-\rho_{m} \varepsilon+\frac{\partial}{\partial x_{j}}\left[\left(\mu+\frac{\mu_{i}}{\sigma_{k}}\right) \frac{\partial k}{\partial x_{j}}\right]
$$

Where $u_{j}$ is the time mean speed.

$$
\begin{gathered}
\frac{\partial\left(\rho_{\mathrm{m}} \varepsilon\right)}{\partial t}+\frac{\partial\left(\rho_{m} u_{j} k\right)}{\partial x_{j}}=C_{\xi 1} \frac{\xi}{k} P_{t}-C_{\xi_{2}} \rho_{m} \frac{\xi^{2}}{k}+\frac{\partial}{\partial x_{j}}\left[\left(\mu+\frac{\mu_{i}}{\sigma_{k}}\right) \frac{\partial \xi}{\partial x_{j}}\right] \\
\mu_{\mathrm{t}}=\frac{C_{\mu} \rho_{m} k^{2}}{\xi}
\end{gathered}
$$

Where $\mu_{t}$ is the turbulent viscosity coefficient, and $P_{t}$ is the generated term of turbulent kinetic energy. In this paper, the model constants are as follows: $C_{\xi 1}=1.44, C_{\xi 2}=1.92$, $\sigma_{\xi}=1.3, \quad \sigma_{\mathrm{k}}=1.0, C_{\mu}=0.09$.

\section{(2) Grid Meshing}

Grid meshing was carried out by unstructured grid. Local grid Meshing encryption was performed at the place where the diffusion angle changed and the position near the water inlet of the pump. The mesh of the lateral inflow forebay was divided into $2,444,900$ nodes, with a total of $13,455,094$ units. The mesh of the front inflow forebay was divided into 3740254 nodes, with a total of 20259141 elements. 


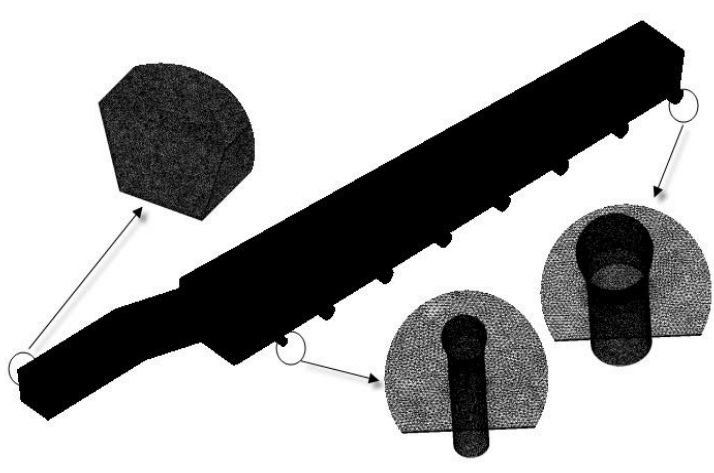

Fig. 3 Grid meshing of structure model of lateral inflow forebay

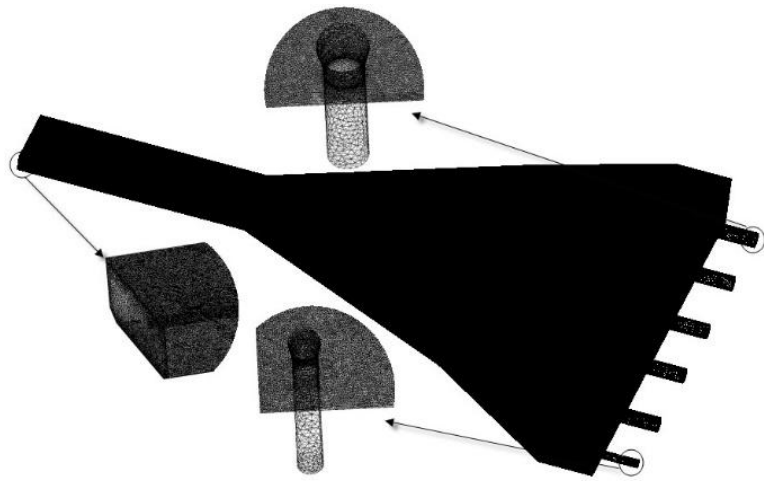

Fig. 4 Grid meshing of structure model of front inflow forebay

In order to ensure the reliability of the calculation results and reduce the calculation workload, the irrelevance analysis of the calculation grid is carried out. Calculate the hydraulic loss from the meshing canal to the inlet pipe of the forward pool and the side forward pool respectively to determine the effective grid number. The calculation formula of hydraulic loss $H_{f}$ is

$$
H_{\mathrm{f}}=\frac{\left(V_{\text {in }}^{2}-V_{\text {out }}^{2}\right)}{2 \mathrm{~g}}+\frac{\left(P_{\text {in }}-P_{\text {out }}\right)}{\rho \mathrm{g}}
$$

Where, $\quad V_{\text {in }}$ Is the average flow velocity at the inlet section; $V_{\text {out }}$ is the average flow velocity at the outlet section; $P_{\text {in }}$ is the inlet pressure; $P_{\text {out }}$ is the outlet pressure.

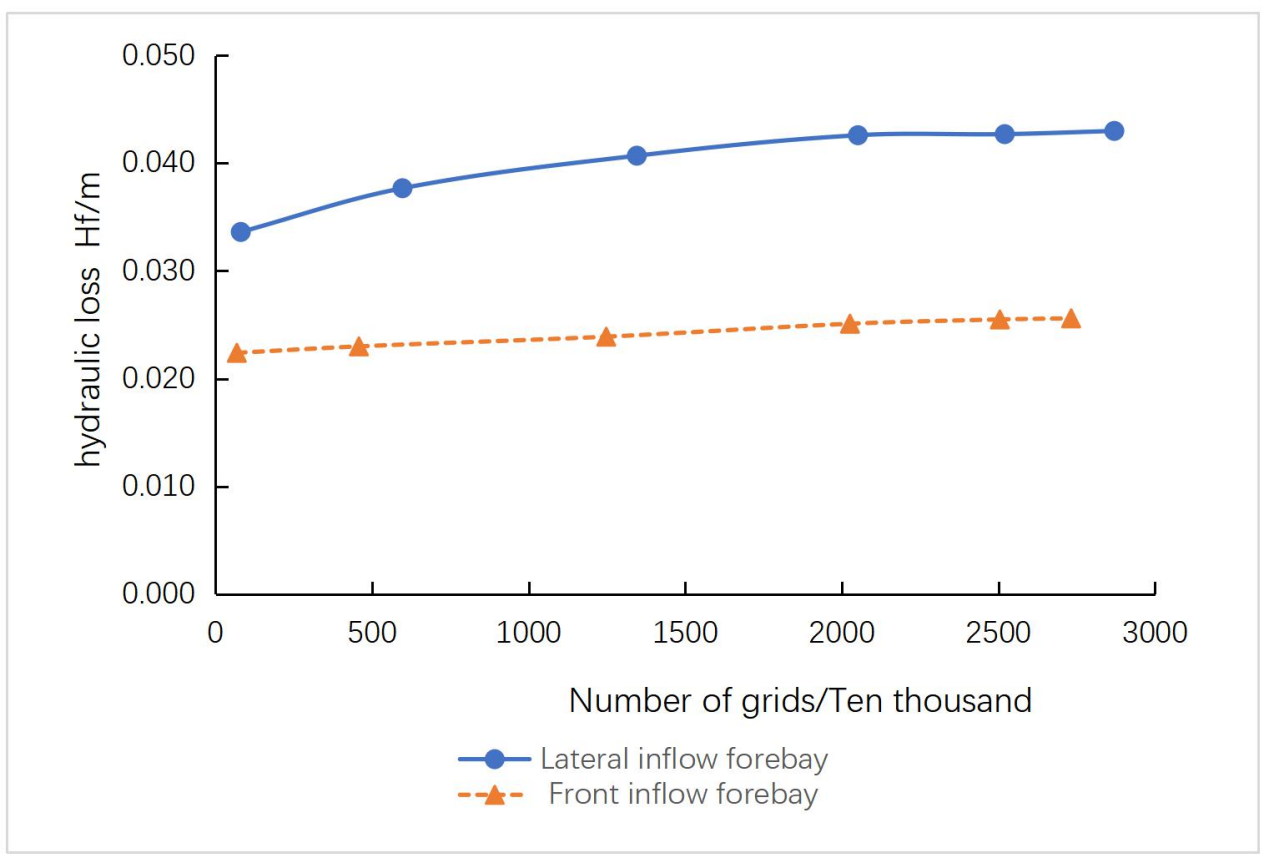

Fig. 4 Verification of meshing independence to simulation results 
The number of grids selected for lateral inflow forebay are 811050, 5973563, 13455994, 20513742, 25200320, 28698507, and the number of grids selected for Front inflow forebay are $27319327,25044832,20259141,12477970,4245825$, and 684591 respectively. The hydraulic loss corresponding to the number of grids lateral inflow forebay and Front inflow forebay is shown in Fig. 5. When the number of grids in the side forward pool and the forward pool is greater than 20 million, the hydraulic loss tends to be stable and there is no significant change. In the end, the number of grids in Front inflow forebay is 20,513,742 and the number of nodes is 3777006; the number of grids in Front inflow forebay is $20,259,141$ and the number of nodes is 3740254 .

\section{(3) Boundary conditions}

In this paper, the boundary conditions of the simulated flow field could be divided into four types, including inlet, wall, outlet and free surface. Among them, the end connected to the intake canal was the inlet boundary; the contact surface between the water flow and the building was the wall boundary; the crossing section of the water intake pipe connected with the pump was the outlet boundary; and the flow wave surface was the free surface boundary. The four types of boundaries were set as: (a) the inlet was set as velocity inlet. The inlet velocity of lateral inflow forebay was set as $0.6087 \mathrm{~m} / \mathrm{s}$, and that of the front inflow forebay was set as $0.6032 \mathrm{~m} / \mathrm{s}$; (b) The outlet boundary was set as the mass flow outlet. In the mass outlet of the lateral inflow forebay, the No. 1 outlet was set as $877.36 \mathrm{~kg} / \mathrm{s}$, and that of the No. $2 \sim 8$ outlets was set as $1244.82 \mathrm{~kg} / \mathrm{s}$. The mass outlet of the positive forward tank is $518.44 \mathrm{~kg} / \mathrm{s}$ at the No. 1 outlet and $1814.54 \mathrm{~kg} / \mathrm{s}$ at the No. $2 \sim 6$ outlets; (c) The wall boundary was set as the non-slip wall, with the absolute roughness of $0.00001 \mathrm{~m}$; (d) In the treating process of the free surface, the heat exchange and conduction of friction between the water surface and air were neglected, and it was set as the rigid cover boundary.

\section{Simulation analysis of flow pattern in the front inflow forebay}

The numerical simulation was conducted on the front inflow forebay and lateral inflow forebay models. After the simulation, a group of sections were selected from the vertical flow direction and along the flow direction, respectively, to analyze the flow on the section and reveal the characteristics of the water flow in the inflow forebay, and then judge the water-energy consumption according to the characteristics of the flow pattern.

The section perpendicular to the water flow direction selected the inlet section of the 
pumping station forebay as the origin. The lateral inflow forebay selected 4 sections in the diversion canal section, with an interval of $9 \mathrm{~m}$. The section of the water intake pool corresponded to the centerline of 8 suction pipes, with a total of 12 sections. The front inflow forebay selected 12 sections, with an interval of $6 \mathrm{~m}$. In the downstream flow direction, the leftmost surface of the pumping station forebay was taken as the origin to be selected to the right in turn. Four sections of the lateral inflow forebay were selected with an interval of $1.92 \mathrm{~m}$. The section of the front inflow forebay was selected to correspond to the center lines of 6 suction pipes, and then one section of the centerline of the forebay was selected, with a total of 7 sections.

Table 1 Parameters of observation section of lateral and front inflow forebays

\begin{tabular}{|c|c|c|c|c|c|c|c|c|c|c|c|c|}
\hline $\begin{array}{l}\text { Vertical flow section of } \\
\text { lateral inflow forebay }\end{array}$ & $\begin{array}{l}y \\
1- \\
1\end{array}$ & $\begin{array}{l}\mathrm{y}_{1} \\
-2\end{array}$ & $\begin{array}{l}\mathrm{y}_{1} \\
-3\end{array}$ & $\begin{array}{l}\mathrm{y}_{1} \\
-4\end{array}$ & $\mathrm{y}_{1-5}$ & $\begin{array}{l}\mathrm{y}_{1-} \\
6\end{array}$ & $\begin{array}{l}\mathrm{y}_{1-} \\
7\end{array}$ & $\begin{array}{l}\mathrm{y}_{1-} \\
8\end{array}$ & $\begin{array}{l}\mathrm{y}_{1-} \\
9\end{array}$ & $\begin{array}{l}\mathrm{y}_{1-} \\
10\end{array}$ & $\begin{array}{l}\mathrm{y}_{1-} \\
11\end{array}$ & $\begin{array}{l}\mathrm{y}_{1-} \\
12\end{array}$ \\
\hline Position of section $(\mathrm{y} / \mathrm{m})$ & 0 & 9 & 18 & 27 & 31.87 & $\begin{array}{l}38 . \\
67\end{array}$ & $\begin{array}{l}47 . \\
67\end{array}$ & $\begin{array}{l}62 . \\
67\end{array}$ & $\begin{array}{l}71 . \\
67\end{array}$ & $\begin{array}{l}80 . \\
67\end{array}$ & $\begin{array}{l}89 . \\
07\end{array}$ & $\begin{array}{l}92 . \\
27\end{array}$ \\
\hline $\begin{array}{l}\text { Vertical flow section of } \\
\text { front inflow forebay }\end{array}$ & $\begin{array}{l}\mathrm{y} \\
2- \\
1\end{array}$ & $\begin{array}{l}\mathrm{y}_{2} \\
-2\end{array}$ & $\begin{array}{l}\mathrm{y}_{2} \\
-3\end{array}$ & $\begin{array}{l}\mathrm{y}_{2} \\
-4\end{array}$ & $\mathrm{y}_{2-5}$ & $\begin{array}{l}y_{2-}- \\
6\end{array}$ & $\begin{array}{l}y_{2-} \\
7\end{array}$ & $\begin{array}{l}\mathrm{y}_{2-} \\
8\end{array}$ & $\begin{array}{l}\mathrm{y}_{2-} \\
9\end{array}$ & $\begin{array}{l}\mathrm{y}_{2-} \\
10\end{array}$ & $\begin{array}{l}\text { Y2- } \\
11\end{array}$ & $\begin{array}{l}\mathrm{y}_{2-} \\
12\end{array}$ \\
\hline Position of section $(\mathrm{y} / \mathrm{m})$ & 0 & 6 & 12 & 18 & 24 & 30 & 36 & 42 & 48 & 54 & 60 & 66 \\
\hline $\begin{array}{l}\text { Concurrent flow section of } \\
\text { lateral inflow forebay }\end{array}$ & $\begin{array}{c}Z_{1} \\
-1\end{array}$ & $\begin{array}{l}\mathrm{Z}_{1-} \\
2\end{array}$ & $\begin{array}{l}Z_{1-} \\
3\end{array}$ & $\begin{array}{l}\mathrm{Z}_{1-} \\
4\end{array}$ & $\begin{array}{l}\text { Concurrent flow section of } \\
\text { front inflow forebay }\end{array}$ & $\mathrm{Z}_{2-1}$ & $\mathrm{Z}_{2-2}$ & $\mathrm{Z}_{2-3}$ & $\mathrm{Z}_{2-4}$ & $\mathrm{Z}_{2-5}$ & $\mathrm{Z}_{2-6}$ & $\mathrm{Z}_{2-7}$ \\
\hline Position of section $(\mathrm{z} / \mathrm{m})$ & 0 & $\begin{array}{l}1 . \\
92\end{array}$ & $\begin{array}{l}3 . \\
84\end{array}$ & $\begin{array}{l}5 . \\
85\end{array}$ & Position of section $(\mathrm{z} / \mathrm{m})$ & 3 & $\begin{array}{l}10 . \\
8\end{array}$ & $\begin{array}{l}20 . \\
35\end{array}$ & $\begin{array}{l}23 . \\
24\end{array}$ & $\begin{array}{l}28 . \\
99\end{array}$ & $\begin{array}{l}38 . \\
34\end{array}$ & $\begin{array}{l}46 . \\
83\end{array}$ \\
\hline
\end{tabular}

\subsection{Uniformity of velocity distribution}

The uniformity of velocity distribution is a physical quantity to judge the flow pattern in the flow field ${ }^{[7 \sim 8]}$. As the flow pattern character in the lateral inflow forebay and front inflow forebay showed certain rules, so a group of sample sections were selected in the lateral inflow forebay pool and the front inflow forebay respectively for comparative analysis.

The sections perpendicular to the water flow direction in the lateral inflow forebay and the front inflow forebay were selected for comparative analysis, and three sample lines were selected on each sample section to represent the flow velocity in the upper, middle and lower part of the forebay, respectively. The height of the surface sample line was $0.5 \mathrm{~m}$ from the free surface, the bottom sample line was $0.5 \mathrm{~m}$ from the bottom floor of the forebay, the middle 
sample line was at a height equal to the bottom and the surface sample line, and 10 sample points were selected at equal distance from the sample line. The uniformity $V_{\text {au }}$ of axial velocity represented the uniformity of axial velocity distribution on the longitudinal section sample line, and its calculation formula is as follows:

$$
V_{\mathrm{au}}=\left[1-\sqrt{\frac{\sum_{\mathrm{i}=1}^{\mathrm{n}}\left(\frac{V_{\mathrm{ai}}}{V_{\mathrm{a}}}-1\right)^{2}}{\mathrm{n}}}\right] \times 100 \%
$$

Where $V_{\text {ai }}$ is the axial velocity $(\mathrm{m} / \mathrm{s})$ corresponding to each grid node of the section; $V_{\mathrm{a}}$ is the average axial velocity of the section $(\mathrm{m} / \mathrm{s}) ; \mathrm{n}$ is the sample size.

According to the location of the section and the selected points, the points on each section were divided into the upper, middle and lower parts. There were 10 points in each section, namely, the normal velocity of 30 points in each section was used as the calculation sample for the uniformity of the section. A total of 360 samples were collected from 12 sections of the lateral inflow forebay and front inflow forebay. Then the simulation results were used to calculate the velocity uniformity at the corresponding positions of the selected sections, and the weighted average was adopted to calculate the weighted average uniformity of the sections. The uniformity results were shown in Table 2 and Table 3, respectively.

Table 2 Calculation results of section velocity uniformity of lateral inflow forebay samples

\begin{tabular}{|c|c|c|c|c|c|c|c|c|c|c|c|c|}
\hline Project & $\mathrm{y}_{1-1}$ & $\mathrm{y}_{1-2}$ & $\mathrm{y}_{1-3}$ & $\mathrm{y}_{1-4}$ & $\mathrm{y}_{1-5}$ & $\mathrm{y}_{1-6}$ & $\mathrm{y}_{1-7}$ & $\mathrm{y}_{1-8}$ & $\mathrm{y}_{1-9}$ & $\mathrm{y}_{1-10}$ & $\mathrm{y}_{1-11}$ & $\mathrm{y}_{1-12}$ \\
\hline $\begin{array}{l}\text { Upper } \\
\text { part }\end{array}$ & 1.000 & 0.999 & 0.998 & 0.984 & -0.279 & 0.581 & 0.477 & 0.600 & 0.471 & 0.474 & 0.307 & 0.312 \\
\hline $\begin{array}{l}\text { Middle } \\
\text { part }\end{array}$ & 1.000 & 0.999 & 0.993 & 0.979 & -0.303 & 0.305 & 0.329 & 0.218 & -0.014 & 0.121 & -0.130 & -0.253 \\
\hline $\begin{array}{l}\text { Lower } \\
\text { part }\end{array}$ & 0.999 & 0.961 & 0.927 & 0.892 & -0.410 & -0.398 & 0.455 & 0.393 & 0.564 & 0.376 & 0.527 & 0.422 \\
\hline $\begin{array}{l}\text { Weighted } \\
\text { average } \\
\text { of } \\
\text { sections }\end{array}$ & 1.000 & 0.986 & 0.973 & 0.952 & -0.331 & 0.163 & 0.420 & 0.404 & 0.340 & 0.323 & 0.235 & 0.160 \\
\hline
\end{tabular}


Table 3 Calculation results of section velocity uniformity of front inflow forebay samples

\begin{tabular}{|c|c|c|c|c|c|c|c|c|c|c|c|c|}
\hline Project & $\mathrm{y}_{2-1}$ & $\mathrm{y}_{2-2}$ & $\mathrm{y}_{2-3}$ & $\mathrm{y}_{2-4}$ & $\mathrm{y}_{2-5}$ & $\mathrm{y}_{2-6}$ & $\mathrm{y}_{2-7}$ & $\mathrm{y}_{2-8}$ & $\mathrm{y}_{2-9}$ & $\mathrm{y}_{2-10}$ & $\mathrm{y}_{2-11}$ & $\mathrm{y}_{2-12}$ \\
\hline Upper part & 1.000 & 0.984 & 0.959 & 0.936 & 0.888 & 0.282 & 0.074 & -0.176 & 0.425 & 0.181 & 0.577 & 0.518 \\
\hline Middle part & 1.000 & 0.990 & 0.972 & 0.960 & 0.952 & 0.286 & -0.038 & -0.165 & -0.311 & 0.296 & 0.330 & 0.130 \\
\hline Lower part & 1.000 & 0.991 & 0.975 & 0.960 & 0.950 & 0.275 & -0.060 & 0.109 & -0.200 & 0.164 & 0.252 & 0.721 \\
\hline $\begin{array}{l}\text { Weighted average } \\
\text { of sections }\end{array}$ & 1.000 & 0.989 & 0.969 & 0.952 & 0.930 & 0.281 & -0.008 & -0.077 & -0.029 & 0.214 & 0.386 & 0.456 \\
\hline
\end{tabular}

According to the uniformity results in Tables 2 and 3, the overall velocity distribution uniformity of the forebay before and after the reconstruction was calculated, respectively. The velocity distribution uniformity of the lateral inflow forebay was $46.9 \%$, and that of the front inflow forebay was $50.5 \%$. The five most uneven sections of the lateral inflow forebay are $\mathrm{y} 1-5, \mathrm{y} 1-12$, and $\mathrm{y} 1-6$, and their mean evenness are $-33.1 \%, 16.0 \%, 16.3 \%$, respectively. The five most uneven sections of the front inflow forebay are y2-8, y2-9, and y2-7, and their average evenness are $-7.7 \%,-2.9 \%$, and $-0.8 \%$, respectively. Obviously, the reflux of the lateral inflow forebay is more obvious than that of the front inflow forebay, and the reflux intensity is larger. Therefore, it could be known that the front inflow forebay had better uniformity and more stable flow pattern distribution than the lateral inflow forebay. In the diversion canal section, the velocity distribution uniformity of both the lateral inflow forebay and front inflow forebay was relatively good, and in the intake canal section, some negative values were produced in the lateral inflow forebay and front inflow forebay, which was because there were two recirculation regions in the flow pattern of the forebay flow, and the flow direction of the recirculation regions was opposite to that of the inlet flow. Therefore, the velocity distribution uniformity was negative, but on the whole, the uniformity of the front inflow forebay =was better than that of the lateral inflow forebay. The higher uniformity of velocity distribution in the front inflow forebay meant that the flow pattern distribution in the front inflow forebay was more stable than that in the lateral inflow forebay, and its turbulence rate was lower and its energy dissipation was less than that in the lateral inflow forebay.

\subsection{Analysis of simulation results of the forebay in vertical flow direction}

The flow pattern simulation results in the vertical flow direction of the lateral inflow forebay are analyzed mainly by the flow pattern distribution of the section in the vertical flow direction. Based on the analysis, the flow pattern in the vertical flow direction of the lateral inflow forebay had the following characteristics: 
The water flow produced vortices in the whole forebay, with a velocity range of $0.01 \sim 1.89 \mathrm{~m} / \mathrm{s}$. It was mainly divided into three sections. The first section was near the inlet, and the vortices were distributed on the sidewalls of both sides of the inlet. Meanwhile, the rotation direction of the vortices was the tangential water flow direction and reversed and pulled forward, with a longer vortex strip. As shown in Figures e $\mathrm{y}_{1-5}$ and $\mathrm{f}_{\mathrm{y}_{1-6}}$, it could be clearly seen that the vortices were generated on both wall sides; The second section was near the middle of the forebay, and the vortex strip was located in the middle and lower part of the water intake forebay. The vortex strip was gradually shortened and the surface velocity was relatively uniform, as shown in Figures $\mathrm{g}_{1-7}$ to $\mathrm{j}_{\mathrm{1} 1-10}$. The third section was the vortex strip at the tail of the forebay, which was mainly distributed on the surface and was interfered by the water reflected from the sidewall. The flow direction of the vortex strip presented periodicity. As shown in Fig. $1 \mathrm{y}_{1-12}$, there were obvious vortices on the water surface.

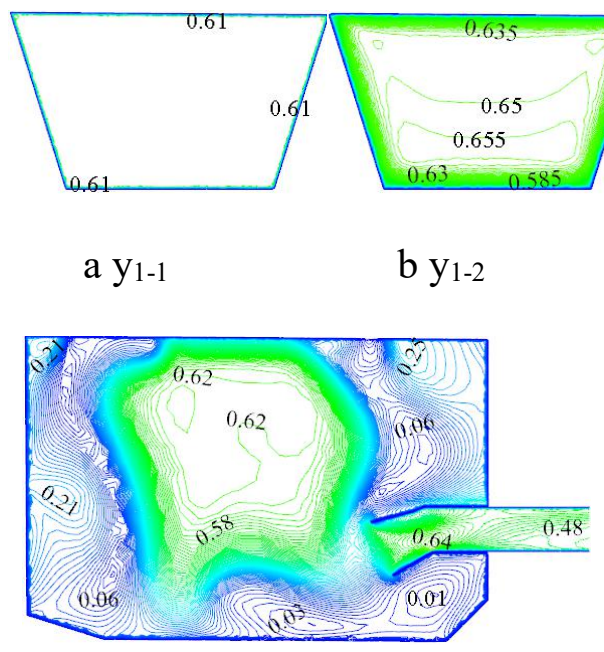

e $y_{1-5}$

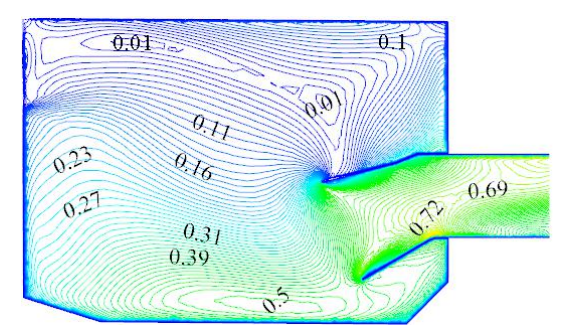

j $\mathrm{y}_{1-7}$

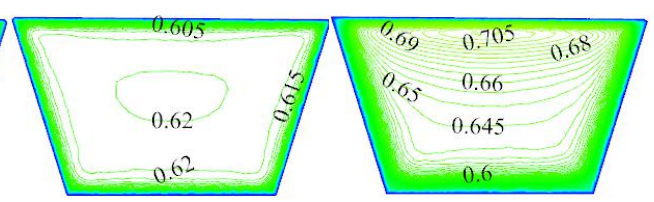

c $\mathrm{y}_{1-3}$

d $\mathrm{y}_{1-4}$

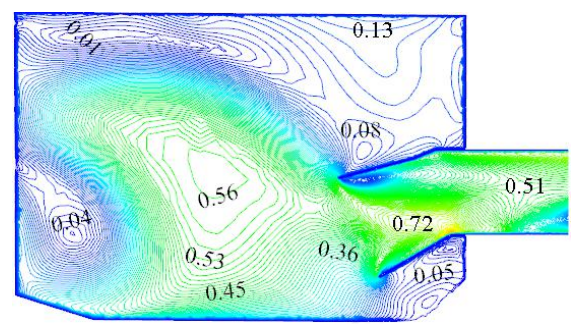

f $y_{1-6}$

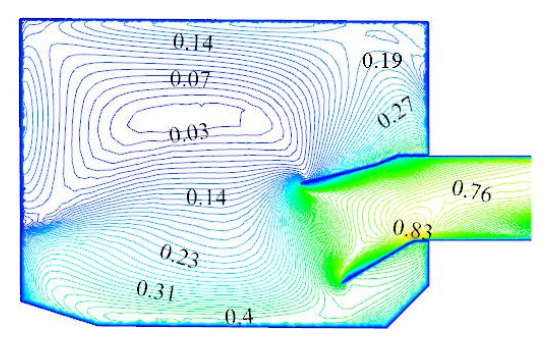

h $\mathrm{y}_{1-8}$ 

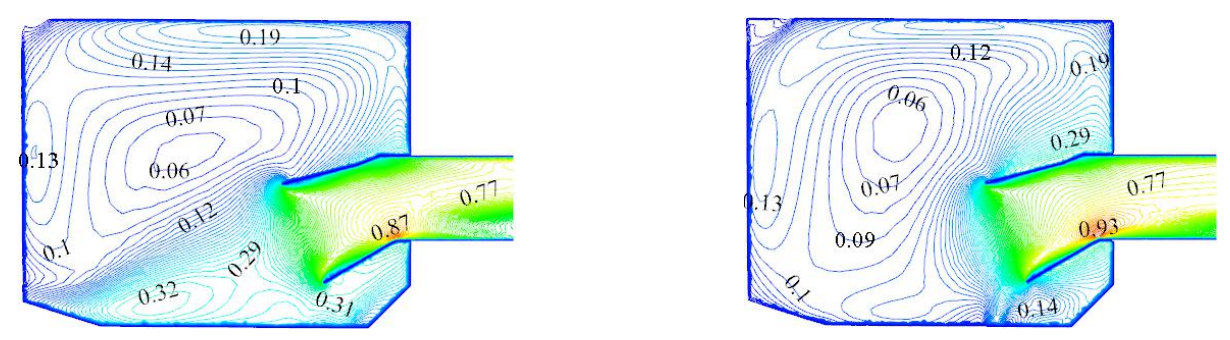

i $\mathrm{y}_{1-9}$

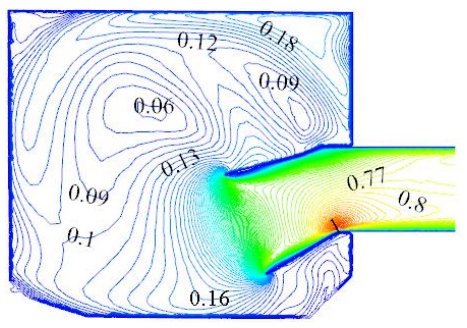

$\mathrm{j} \mathrm{y}_{1-10}$

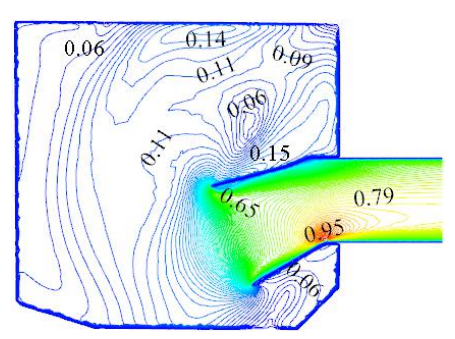

$\mathrm{k} \mathrm{y}_{1-11}$

$1 \mathrm{y}_{1-12}$

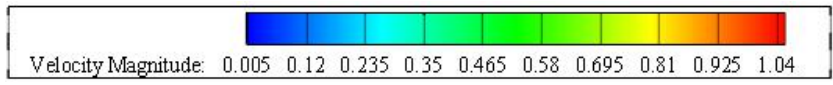

Fig. 5 Contour map of velocity distribution on the section perpendicular to the flow direction of the lateral inflow forebay

The flow pattern simulation results in the vertical flow direction of the forebay were analyzed mainly by the flow pattern distribution of the section perpendicular to the flow direction. Based on the analysis, the flow pattern in the direction perpendicular to the forebay had the following characteristics:

First of all, the velocity distribution characteristics showed good symmetry. The water flow in the forebay was accelerated, with a velocity range of $0.005 \sim 0.865 \mathrm{~m} / \mathrm{s}$. Secondly, along the water flow direction, the velocity distribution was related to the shape of the section. The velocity distribution at the inlet showed that the water flow was less affected by the water reflected back by the sidewall and the velocity distribution was more uniform. The closer the flow was to the sidewall, the greater the disturbance of the velocity would be. Thirdly, it could be seen from the velocity distribution on several sections at the front of the sidewall where the suction pipe mouth was placed that although the flow pattern distribution was still symmetrical, there was flow with mutual interference of vortices, and the upper disturbance was larger than the lower one, as shown in Figs. $\mathrm{k}_{2-11}$ and $1 \mathrm{y}_{2-12}$ 


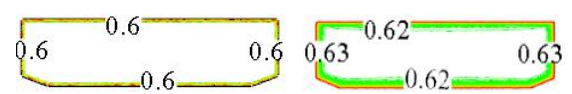

a y2-1

b y2-2

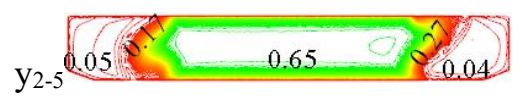

f $y_{2}-6$

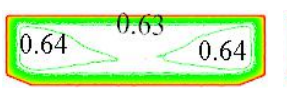

c y2-3

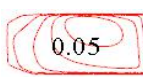

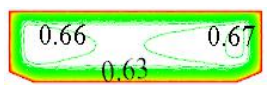

d y2-4

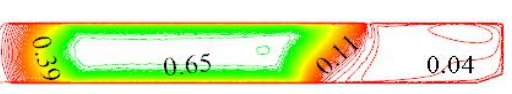

g $\mathrm{y}_{2-7}$

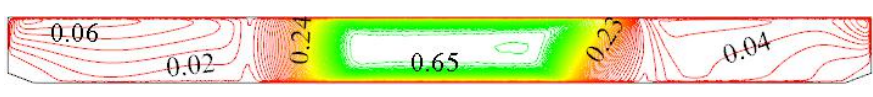

h $\mathrm{y}_{2-8}$

0.06 0.04 0.02 is $\quad 0.67 \quad 0.003$

i y2-9

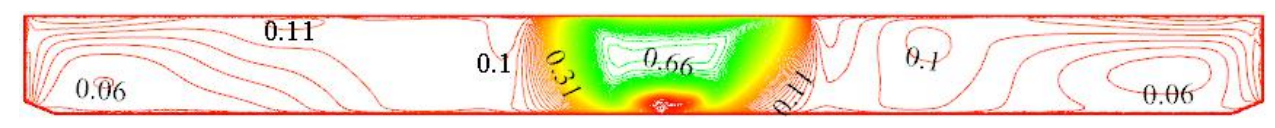

j y2-10

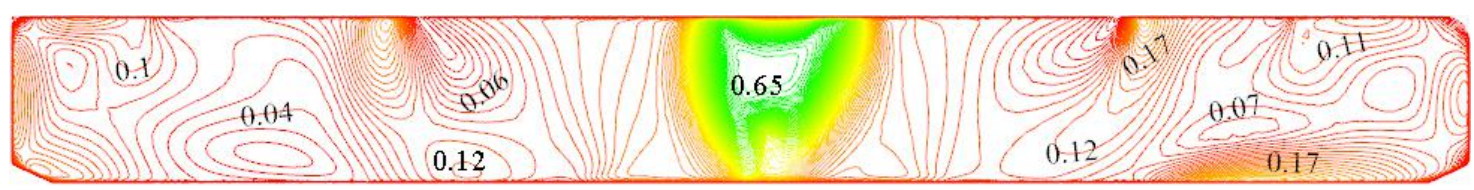

k y2-11

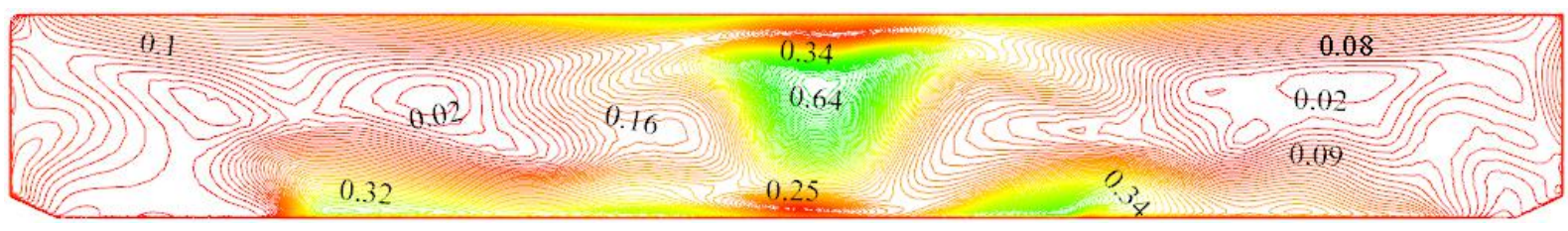

$1 \mathrm{y}_{2-12}$

Velocity Magnitude: $\quad 0.01 \quad 0.12 \quad 0.23 \quad 0.340 .450 .560 .67 \quad 0.780 .89 \quad 1 \quad 1.11$

Fig. 6 Contour map of velocity distribution on the section perpendicular to the flow direction of the front inflow forebay

\subsection{Analysis of simulation results of the forebay in downstream direction}

The fluid simulation results of the downstream flow direction of the lateral inflow forebay were analyzed mainly by the flow pattern distribution of the downstream flow direction section. Based on the analysis, the flow pattern in the downstream direction of the lateral inflow forebay had the following characteristics: 
(1) The flow in the downstream flow direction was not smooth, with a flow velocity ranging from 0.05 to $1.85 \mathrm{~m} / \mathrm{s}$. The vortices with a certain speed appeared at the corresponding positions of the suction pipe orientations of each pump, and the position of the vortices should deviate from the right front of the water intake pipe mouth, as shown in Fig. $\mathrm{d} \mathrm{z}_{1-4}$. (2) The vortices also appeared in the middle position of the water inlets of the two pumps, and the main direction was flowing forward along the flow direction, with a longer vortex strip. The length of the vortex strip was shortened and widened along the flow direction, and rotated around, and then the vortex strip and the water flow entering the suction pipe mouth formed a more obviously tangential rotation state, thus generating a new vortex strip. This vortex band expanded in the right front of the suction pipe mouth and extended to the surface of the water flow. The vortex strip generated around the suction pipe mouth decreased along the width direction of the lateral inflow forebay, which could be clearly seen in Figures $b_{z_{1-2}}$ and $\mathrm{c}_{1-3}$.

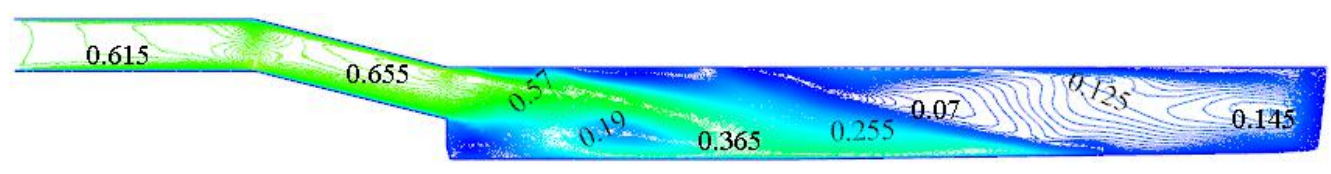

a $\mathrm{Z}_{1-1}$

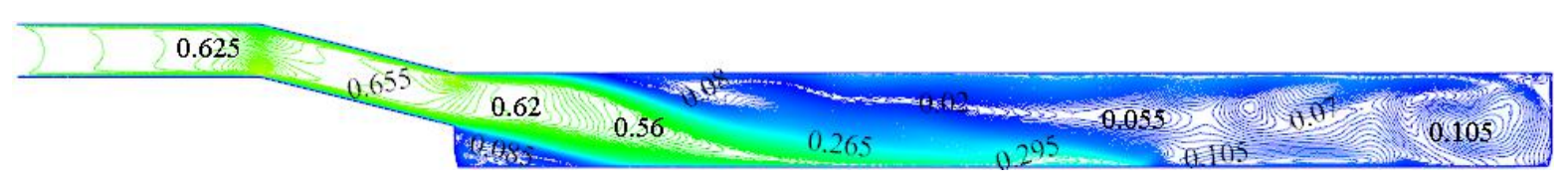

b $\mathrm{Z}_{1-2}$

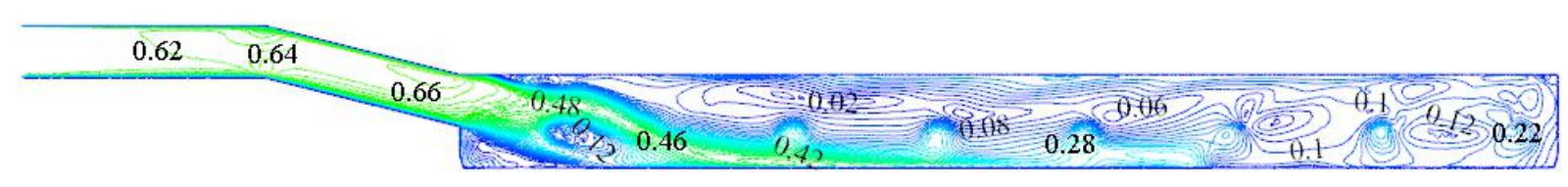

c $\mathrm{Z}_{1-3}$

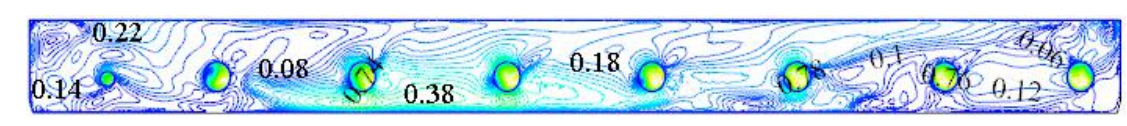

d $Z_{1-4}$

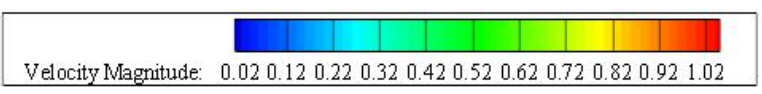

Fig. 7 Contour map of sectional velocity distribution on the section along the flow direction of the lateral inflow forebay

The simulation results of the flow pattern along the flow direction of the front inflow forebay 
were analyzed mainly by the flow pattern distribution along the flow direction. Based on the analysis, the flow pattern parallel to the water flow direction of the lateral inflow forebay had the following characteristics:

(1) Along the water flow direction, the water flow pattern was uniform, and the water flow was accelerated on the whole. The flow velocity ranged from $0.05 \mathrm{~m} / \mathrm{s}$ to $1.23 \mathrm{~m} / \mathrm{s}$. Only a part of the flow vortex belt appeared near the front end of the sidewall where the suction pipe was placed, and the vortex belt presented downward rotation, while the velocity in the middle presented a high-speed zone. The upper water flow was tangent between the water reflected back from the sidewall of the suction pipe and the water flow in the forebay, and there were low-speed vortex zones distributed. (2) The water flow on both sides of the forebay presented current vortexes, whose rotation direction pointed to the middle, forming a small vortex belt with internal rotation. The velocity of the vortex belt was small, and the region was along the sidewall. As shown in Figs. a $\mathrm{z}_{2-1}$ and $\mathrm{g}_{2} \mathrm{y}_{2-7}$, it could be seen that the flow velocity of the vortex belt was $0.01 \sim 0.04 \mathrm{~m} / \mathrm{s}$; (3) When the water flowed through the inlet of the suction pipe, the velocity distribution was more uniform, and the flow vortex zone near the front end of the sidewall where the suction pipe was placed decreased, which was conducive to the intake of water from the suction pipe, and then the velocity distribution features of downward flow appeared on the water above the inlet of the suction pipe.

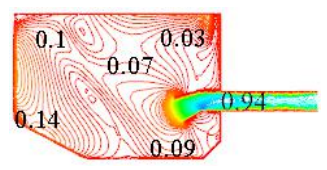

a $Z_{2-1}$

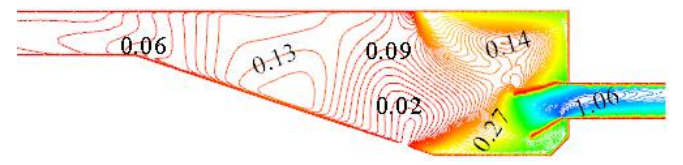

c $Z_{2-3}$

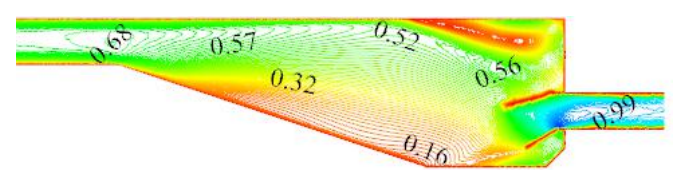

e $Z_{2-5}$

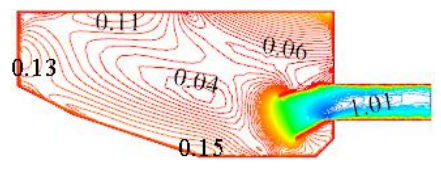

g Z2-7

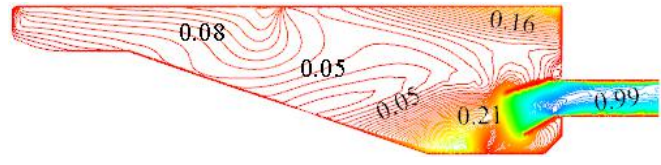

b z $2-2$
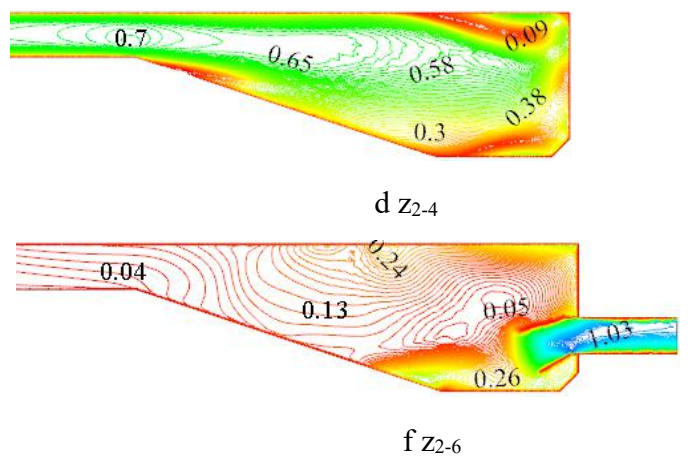
Fig. 8 Contour map of sectional velocity distribution on the section along the flow direction of the front inflow forebay

\subsection{Comprehensive analysis}

According to the analysis of the simulation results, it could be concluded that on the section along the direction of the water flow, the water flow was smooth at the inlet of the suction pipe of the front inflow forebay, while vortices were generated at the suction pipe of the lateral inflow forebay. The overall water flow of the front inflow forebay was fluent and smooth, while the water flow of the lateral inflow forebay wasn't fluent. In the direction of the vertical water flow, the velocity distribution characteristics of the front inflow forebay showed good symmetry, and the water flow in the front inflow forebay showed the characteristics of accelerated flow. The flow velocity distribution along the water flowing direction was related to the shape of the section, while the water flow of the lateral inflow forebay had vortices in the whole forebay. The eddies were generated near the suction pipe at the outlet of the flow field, and the velocity distribution at the outlet of the front inflow forebay still showed good symmetry. The eddies were in front of the inlet of the suction pipe, and the velocity accelerated from the surrounding to the inlet of the suction pipe, as shown in Fig. 10. Besides, along the water flowing direction of the lateral inflow forebay, the eddies also appeared in the middle of the intake pipe orifice of the two pumps, causing disturbance to the water flow, as shown in Fig. 9. According to the flow pattern analysis results of the vertical flow direction and the flow direction of the forebay before and after the reconstruction, the flow pattern distribution characteristics of the front inflow forebay were more stable than those of the lateral inflow forebay, with fewer eddies and lower water energy consumption. 


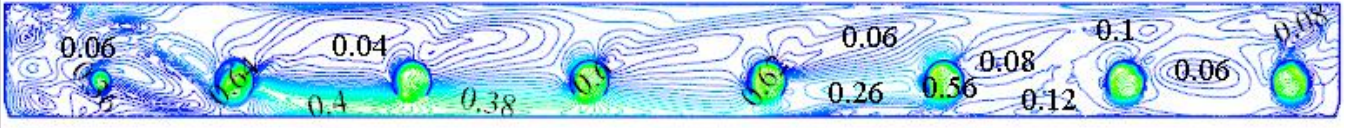

Fig. 9 Flow velocity diagram of the section at the exit of the lateral inflow forebay

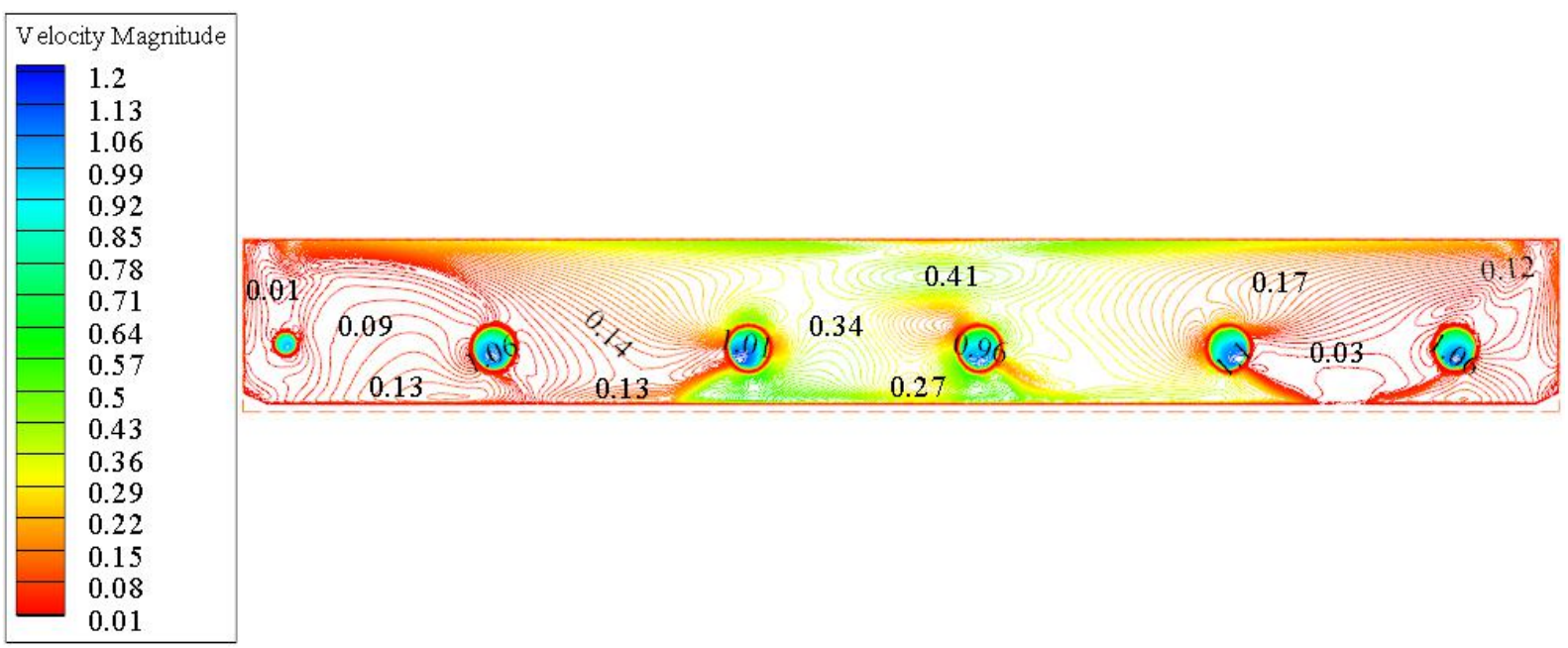

Fig. 10 Flow velocity diagram of the section at the exit of the front inflow forebay

\subsection{Calculation of energy consumption rate}

The energy consumption rate per unit volume of water flow in the front inflow and lateral inflow forebays was calculated to identify the energy consumption loss. The so-called energy consumption rate per unit volume of water is the energy loss per unit time of a unit volume of water ${ }^{[9-10]}$. The flow of each unit was set as $Q_{\mathrm{i}}$, the velocity as $\mathrm{u}_{i}$, the volume of water in the unit body as $V_{\mathrm{i}}$, and the water level corresponding to the center point of the grid of the unit body as $z_{i}$. Then selecting all the time that the water flowed through the front inflow forebay as the whole calculation period, the water-energy consumption rate per unit volume of the system in each $\Delta \mathrm{t}$ time was calculated as follows: 


$$
\Phi_{V}=\frac{\left(E_{\mathrm{t}}+E_{\mathrm{u}}-E_{\mathrm{d}}\right)-E_{\mathrm{t}+\Delta \mathrm{t}}}{\frac{1}{2}\left(V_{\mathrm{t}}+V_{\mathrm{t}+\Delta \mathrm{t}}\right) \Delta \mathrm{t}}
$$

Where $\Phi_{V}$ is the energy consumption rate per unit volume of fluid; The energy at the initial moment is $E_{\mathrm{t}}$, and the volume of water is $E_{\mathrm{t}}$; After time $\Delta \mathrm{t}$, the energy is $E_{\mathrm{t}+\Delta \mathrm{t}}$, and water volume is $V_{\mathrm{t}+\Delta \mathrm{t}}$; Within the time $\Delta \mathrm{t}$, the input energy of the inlet section is $E_{\mathrm{u}}$, and the output energy of the outlet section is $E_{\mathrm{d}} ; V_{\mathrm{t}}=V_{\mathrm{t}+\Delta \mathrm{t}}=\sum V_{\mathrm{i}} ; E_{\mathrm{t}}=E_{\mathrm{t}+\Delta \mathrm{t}}=\sum E_{\mathrm{i}} ; E_{\mathrm{i}}=\left(\mathrm{z}_{\mathrm{i}}+\frac{\mathrm{u}_{\mathrm{i}}^{2}}{2 \mathrm{~g}}\right) \rho \mathrm{g} V_{\mathrm{i}} ; E_{\mathrm{u}}=E_{\mathrm{d}}=\sum E_{\mathrm{j}}$; $E_{\mathrm{j}}=\left(\mathrm{z}_{\mathrm{i}}+\frac{\mathrm{u}_{\mathrm{i}}^{2}}{2 \mathrm{~g}}\right) \rho \mathrm{g} Q_{\mathrm{i}} \Delta \mathrm{t}$

Using the simulation results of $100 \mathrm{~s}$, the changing relationship of energy consumption with time before and after the reconstruction of the pumping station forebay was calculated. As shown in Fig. 11, the energy consumption gradually decreased with the running time, from $5851.11 \mathrm{~W} / \mathrm{m}^{2}$ to $608.13 \mathrm{~W} / \mathrm{m}^{2}$ in the lateral inflow forebay and from $4951.25 \mathrm{~W} / \mathrm{m}^{2}$ to $492.81 \mathrm{~W} / \mathrm{m}^{2}$ in the front inflow forebay. Finally, it tended to be stable, and the lateral energy consumption was higher than the front energy consumption.

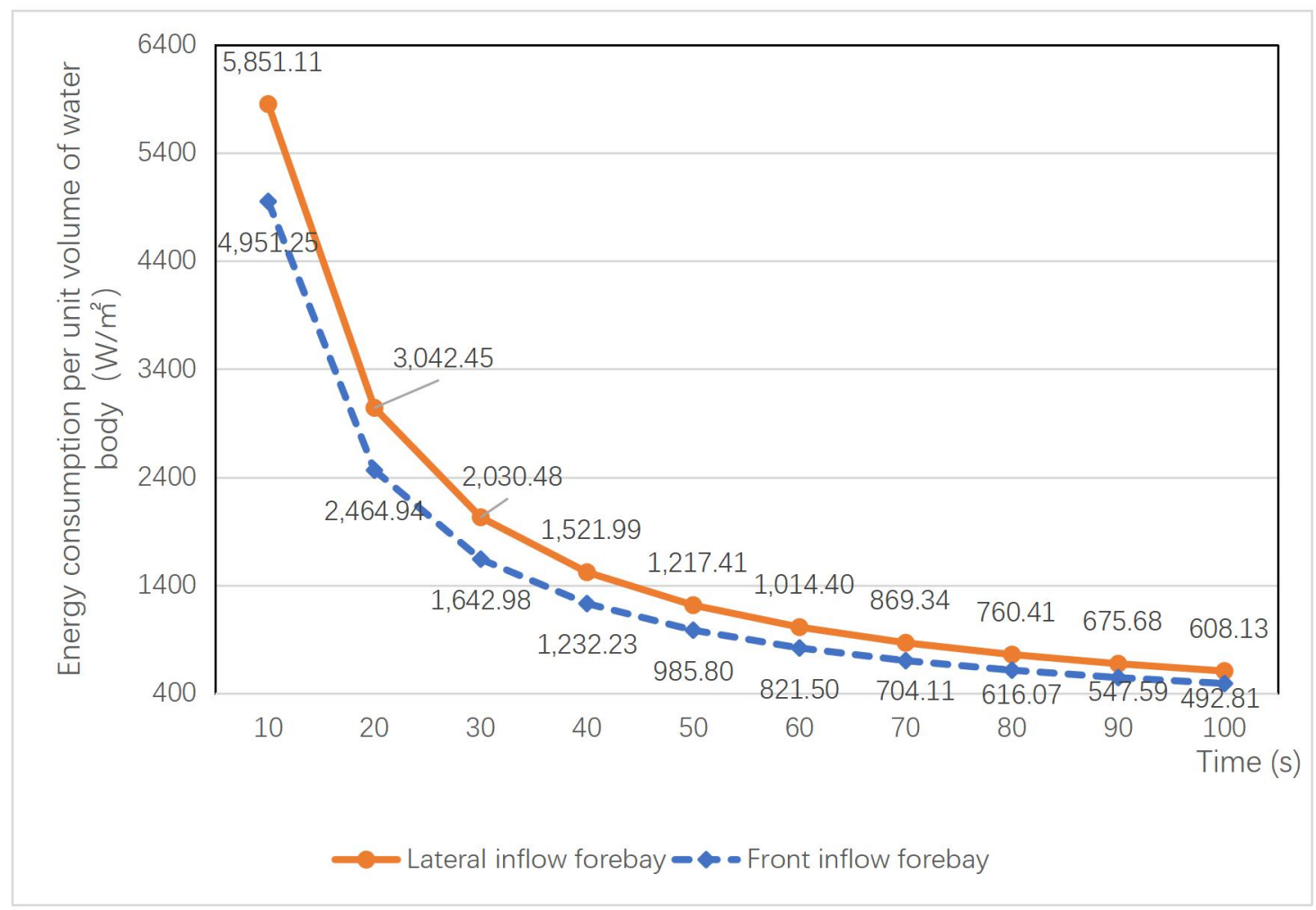

Fig. 11 Trend of water body energy dissipation rate per unit volume in the pumping station forebay with time

As shown in Fig. 11, after comparing the changes of water energy consumption per unit volume of the front inflow forebay and lateral inflow forebay with time, it was found that the 
energy consumption per unit volume of the two decreased progressively with the time, but during the process, the energy consumption per unit volume of the front inflow forebay continued to be smaller than that of the lateral inflow forebay. Therefore, the energy loss of the front inflow forebay was lower than that of the lateral inflow forebay during the operation of the pumping station.

\section{Conclusion}

Based on the comparative analysis of the flow pattern characteristics of the pumping station forebay before and after the reconstruction, the following conclusions were drawn:

(1) By comparing the simulation results of the pumping station forebay before and after the reconstruction, it could be concluded that the flow pattern characteristics in the front inflow forebay were more stable than those in the lateral inflow forebay, and the flow pattern characteristics in the front inflow forebay had good symmetry. At the same time, the uniformity in the water flowing process was better, and the velocity distribution uniformity in the front inflow forebay was better than that in the lateral inflow forebay.

(2) The flow pattern characteristics of the pumping station forebay were associated with energy consumption, and the flow pattern characteristics could be solved by numerical simulation to analyze the energy consumption changes. According to the simulation results, the overall energy dissipation rate of the front inflow forebay was lower than that of the lateral inflow forebay.

(3) The energy dissipation in the pumping station forebay could be reflected by the turbulence situation, and the changes in the shape and size of the pumping station forebay had an impact on the loss of energy consumption. In the water flowing process, there were fewer vortices in the front inflow forebay than in the lateral inflow forebay, with a good energy-saving effect. 


\section{References}

[1] Qiang Changlin, Sun Yuhua. Exploration and Practice of Energy Conservation and Consumption Reduction in Booster Pump Station of City Water Supply[J]. China Water \& Wastewater, 2013, 29(2):16-18. (in Chinese)

[2] $\mathrm{Xu}$ Bo, Gao Chen, Xia Hui. Influence of Geometric Parameters of Open Diversion Piers on Modifying Effect of Sluice Gate Station [J]. Journal of Yangtze River Scientific Research Institute, 2019, 36(02):58-62. (in Chinese)

[3] Jiang Wen, Yu Jianzhong, Fu Zongfu. Flow Pattern and Modifying Measures of the Pumping Station Inflow Forebay in Downstream of the Bend Reach [J]. Yellow River, 2019, 41(04):83-87+118. (in Chinese)

[4] Tong Hongwei, Wang Xiaisheng. Research on Improvement Measures of Regulating Reservoir Flow Pattern in Urban Pumping Station [J]. China Rural Water and Hydropower, 2018(2):136-139. (in Chinese)

[5] Bai Yuchuan, Li Bin, Xu Haijue. Analysis of the Flow Pattern Modifying Measures of Multiple Herringbone Forebay in Large Span Pumping Station [J]. Water and Power Energy Science, 2019,37(06):75-79. (in Chinese)

[6] Han Feng, Liang Jindong. Numerical Simulation and Improvement of Flow Pattern of Unilateral Inflow Forebay in Pumping Station [J]. China Rural Water and Hydropower,2018(08):190-193. (in Chinese)

[7] Zhao Haoru, Yang Fan, Liu Chao. Research on Flow Pattern Simulation and Improvement of Lateral Inflow Pump Station [J]. Water Resources and Hydropower Technology, 2017,48(07):79-84. (in Chinese)

[8] Luo Can, Liu Chao. Research on Simulation and Improvement of Lateral Inflow Characteristics of Multi-Unit Pump Station [J]. Journal of Hydroelectric Engineering, 2015,34(01):207-214. (in Chinese)

[9] Shi Wei, Cheng Li. Numerical Simulation and Improvement of Water Flow Pattern of Water Intake Pumping Station in Water Source [J/OL]. South-North Water Transfer and Water Science and Technology :1-31[2020-05-09]. (in Chinese)

[10] Chang Mei, Xu Guobin. Numerical Tank Simulation and Verification of the Principle of Minimum Energy Consumption Rate [J]. Journal of Sediment Research, 2013(02):67-71. (in Chinese) 